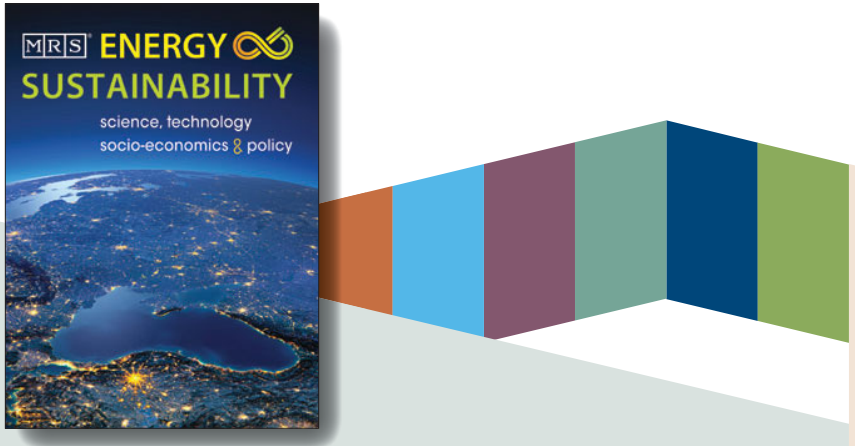

Key issues for Li-ion battery recycling

Linda Gaines, Kirti Richa, Jeffrey Spangenberger

Lithium-ion batteries (LIBs) have gained popularity because of their high energy and power density and long cycle life. But rapid adoption brings with it the challenge of end-of-life management. The authors build a strong case as to why LIBs should be recycled and address the different factors affecting LIB recycling.

https://doi.org/10.1557/mre.2018.13

\section{Rare earths: A review of the landscape}

Rajive Ganguli, Douglas R. Cook

Rare earths (REE) are critical components to many technologies that drive the modern world. Can recycling and substitution make a dent in the demand for REE in the near future? Though present in most parts of the world, they are produced mostly in China. The authors review various aspects of rare earths including extraction, geopolitics, and challenges.

https://doi.org/10.1557/mre.2018.7

Think circular-Reducing embodied carbon

through materials selection

Fiona Cousins, Tiffany Broyles Yost, Gray Bender

As the operational carbon in the built environment falls through improved design, the embodied carbon released from extraction, manufacturing, and transportation of materials becomes more significant. The authors explain how material choices can affect environmental conditions and human health, and showcase how decision making can be improved through greater transparency and a broader view of materials impact.

https://doi.org/10.1557/mre.2018.3

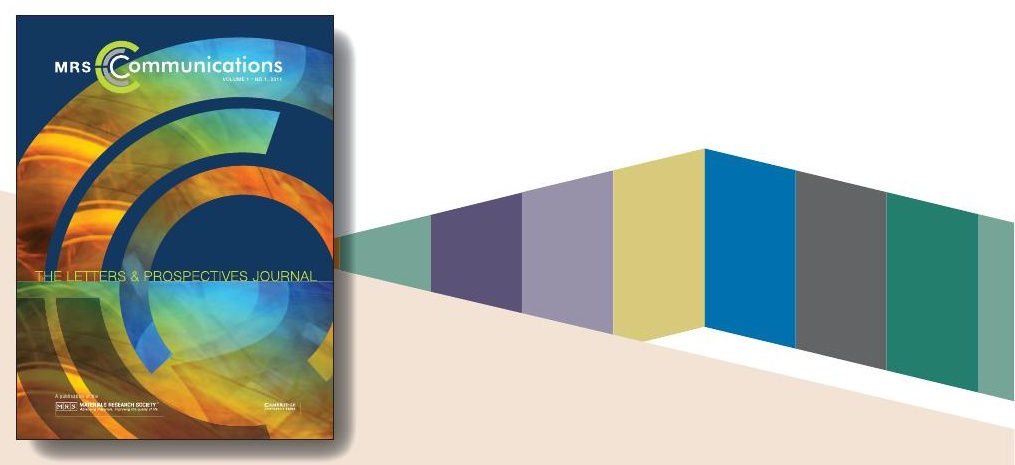

Piezoelectric bioMEMS cantilever for measurement of muscle contraction and for actuation of mechanosensitive cells

Elizabeth A. Coln, Alisha Colon, Christopher J. Long, Narasimhan Narasimhan Sriram, Mandy Esch, Jean-Matthieu Prot, Daniel H. Elbrecht, Ying Wang, Max Jackson, Michael L. Shuler, James J. Hickman

Actuator devices and cantilevers that enable sensitive and selective responses with cells such as myocites and cardiomyocites are a breakthrough in the quantitative investigation and stimuli-responsive properties for bioMEMs devices. The piezoelectric response is a way to improve this quantification and possible tissue health monitoring applications. https://doi.org/10.1557/mrc.2019.129

\section{Enhancing ionic conductivity with fluorination in organosilyl solvents for lithium-ion battery electrolytes}

Leslie J. Lyons, Tom Derrah, Steven Sharpe, Seiyoung Yoon, Scott Beecher, Monica Usrey, Adrián Peña-Hueso, Tobias Johnson, Robert West

The ability to enhance ionic conductivity in lithium battery operation with smart electrolytes improves the efficiency, capacity, and supercapacity properties of charging devices. The fluoroorganosilyl (FOS) modification offers a pathway to improve these properties by altering the salt-dissociation equilibrium. It is possible to explore other series of perfluorostructures and surfactants.

https://doi.org/10.1557/mrc.2019.131

\section{Electrospinning deposition of poly(acrylic acid):} Platinum/carbon catalyst ink to enhance polymer electrolyte membrane fuel-cell performance

\section{Guan Hao Chen, Danielle Kelly, Audrey Shine, Zipei Liu,} Likun Wang, Stoyan Bliznakov, Miriam Rafailovich

The authors highlight the use of polymeric hosts accessed by electrospinning and their ability to be used more effectively in fuelcell catalysts. The approach makes use of the temperature-dependent deformation properties to attribute the thermomechanical properties with the composition and method of fiber formation.

https://doi.org/10.1557/mrc.2019.144 